\section{OPEN ACCESS}

Edited by:

Jiapeng Huang,

University of Louisville, United States

Reviewed by:

Abele Donati,

Marche Polytechnic University, Italy

Eizo Watanabe,

Chiba University, Japan

*Correspondence:

Faeq Husain-Syed

faeq.husain-syed@

innere.med.uni-giessen.de

István Vadász

istvan.vadasz@

innere.med.uni-giessen.de

Specialty section:

This article was submitted to Intensive Care Medicine and

Anesthesiology,

a section of the journal

Frontiers in Medicine

Received: 24 August 2020 Accepted: 07 October 2020

Published: 12 November 2020

Citation:

Husain-Syed F, Birk H-W, Wilhelm J,

Ronco C, Ranieri VM, Karle B, Kuhnert S, Tello K, Hecker M,

Morty RE, Herold S, Kehl O,

Walmrath H-D, Seeger W and Vadász I (2020) Extracorporeal Carbon

Dioxide Removal Using a Renal

Replacement Therapy Platform to Enhance Lung-Protective Ventilation in

Hypercapnic Patients With Coronavirus Disease 2019-Associated Acute Respiratory Distress Syndrome.

Front. Med. 7:598379

doi: 10.3389/fmed.2020.598379

\title{
Extracorporeal Carbon Dioxide
} Removal Using a Renal Replacement Therapy Platform to Enhance Lung-Protective Ventilation in Hypercapnic Patients With Coronavirus Disease 2019-Associated Acute Respiratory Distress Syndrome

Faeq Husain-Syed ${ }^{1,2,3 *}$, Horst-Walter Birk ${ }^{1}$, Jochen Wilhelm ${ }^{2,4,5,6}$, Claudio Ronco ${ }^{3,7}$, V. Marco Ranieri ${ }^{8}$, Bianka Karle ${ }^{1}$, Stefan Kuhnert' ${ }^{2}$ Khodr Tello ${ }^{2,4,5}$, Matthias Hecker ${ }^{2,4}$, Rory E. Morty ${ }^{2,4,5,9}$, Susanne Herold ${ }^{2,4,5}$, Oliver Kehl ${ }^{1}$, Hans-Dieter Walmrath ${ }^{1,2}$, Werner Seeger ${ }^{1,2,4,5,6,9}$ and István Vadász ${ }^{2,4,5 *}$

${ }^{1}$ Divison of Nephrology, Department of Internal Medicine II, University Hospital Giessen and Marburg, Justus Liebig University Giessen, Giessen, Germany, ${ }^{2}$ Division of Pulmonary and Critical Care Medicine, Department of Internal Medicine II, University Hospital Giessen and Marburg, Justus Liebig University Giessen, Giessen, Germany, ${ }^{3}$ International Renal Research Institute of Vicenza, San Bortolo Hospital, Vicenza, Italy, ${ }^{4}$ Universities of Giessen and Marburg Lung Center, Justus Liebig University Giessen, Giessen, Germany, ${ }^{5}$ The Cardio-Pulmonary Institute, Giessen, Germany, ${ }^{6}$ Institute for Lung Health, Justus Liebig University Giessen, Giessen, Germany, ${ }^{7}$ Department of Medicine (DIMED), Università di Padova, Padua, Italy, ${ }^{8}$ Department of Medical and Surgical Sciences (DIMEC), Anaesthesia and Intensive Care Medicine, Sant'Orsola-Malpighi Hospital, Alma Mater Studiorum University of Bologna, Bologna, Italy, ${ }^{9}$ Department of Lung Development and Remodelling, Max Planck Institute for Heart and Lung Research, Bad Nauheim, Germany

Coronavirus disease 2019 (COVID-19)-associated acute respiratory distress syndrome (ARDS) is associated with high mortality. Lung-protective ventilation is the current standard of care in patients with ARDS, but it might lead to hypercapnia, which is independently associated with worse outcomes. Extracorporeal carbon dioxide removal $\left(\mathrm{ECCO}_{2} \mathrm{R}\right)$ has been proposed as an adjuvant therapy to avoid progression of clinical severity and limit further ventilator-induced lung injury, but its use in COVID-19 has not been described yet. Acute kidney injury requiring renal replacement therapy (RRT) is common among critically ill COVID-19 patients. In centers with available dialysis, low-flow $\mathrm{ECCO}_{2} \mathrm{R}$ ( $<500 \mathrm{~mL} / \mathrm{min}$ ) using RRT platforms could be carried out by dialysis specialists and might be an option to efficiently allocate resources during the COVID-19 pandemic for patients with hypercapnia as the main indication. Here, we report the feasibility, safety, and efficacy of $\mathrm{ECCO}_{2} \mathrm{R}$ using an RRT platform to provide either standalone $\mathrm{ECCO}_{2} \mathrm{R}$ or $\mathrm{ECCO}_{2} \mathrm{R}$ combined with RRT in four hypercapnic patients with moderate ARDS. A randomized clinical trial is required to assess the overall benefit and harm.

Clinical Trial Registration: ClinicalTrials.gov. Unique identifier: NCT04351906.

Keywords: continuous renal replacement therapy, respiratory acidosis, SARS-CoV-2, extracorporeal organ support, respiratory dialysis 


\section{INTRODUCTION}

The percentages of coronavirus disease 2019 (COVID-19) patients diagnosed with acute respiratory distress syndrome (ARDS) range between 20 and $67 \%$ of hospitalized patients $(1,2)$ and $100 \%$ of mechanically ventilated patients (3) and are associated with high mortality (2). Lung-protective ventilation is the current standard of care for ARDS (4), which limits ventilator-induced lung injury but may lead to elevated carbon dioxide $\left(\mathrm{CO}_{2}\right)$ levels and respiratory acidosis, which are independently associated with worse outcomes in the setting of $\operatorname{ARDS}(5,6)$. In these patients, extracorporeal $\mathrm{CO}_{2}$ removal $\left(\mathrm{ECCO}_{2} \mathrm{R}\right)$ may help to avoid the progression of clinical severity (5). Acute kidney injury (AKI) is common among critically ill COVID-19 patients, with $\sim 20 \%$ requiring renal replacement therapy (RRT) (7). Recent studies have proposed the integration of $\mathrm{ECCO}_{2} \mathrm{R}$ into continuous RRT (CRRT) platforms to provide combined $\mathrm{CO}_{2}$ removal and renal support using low blood-flow

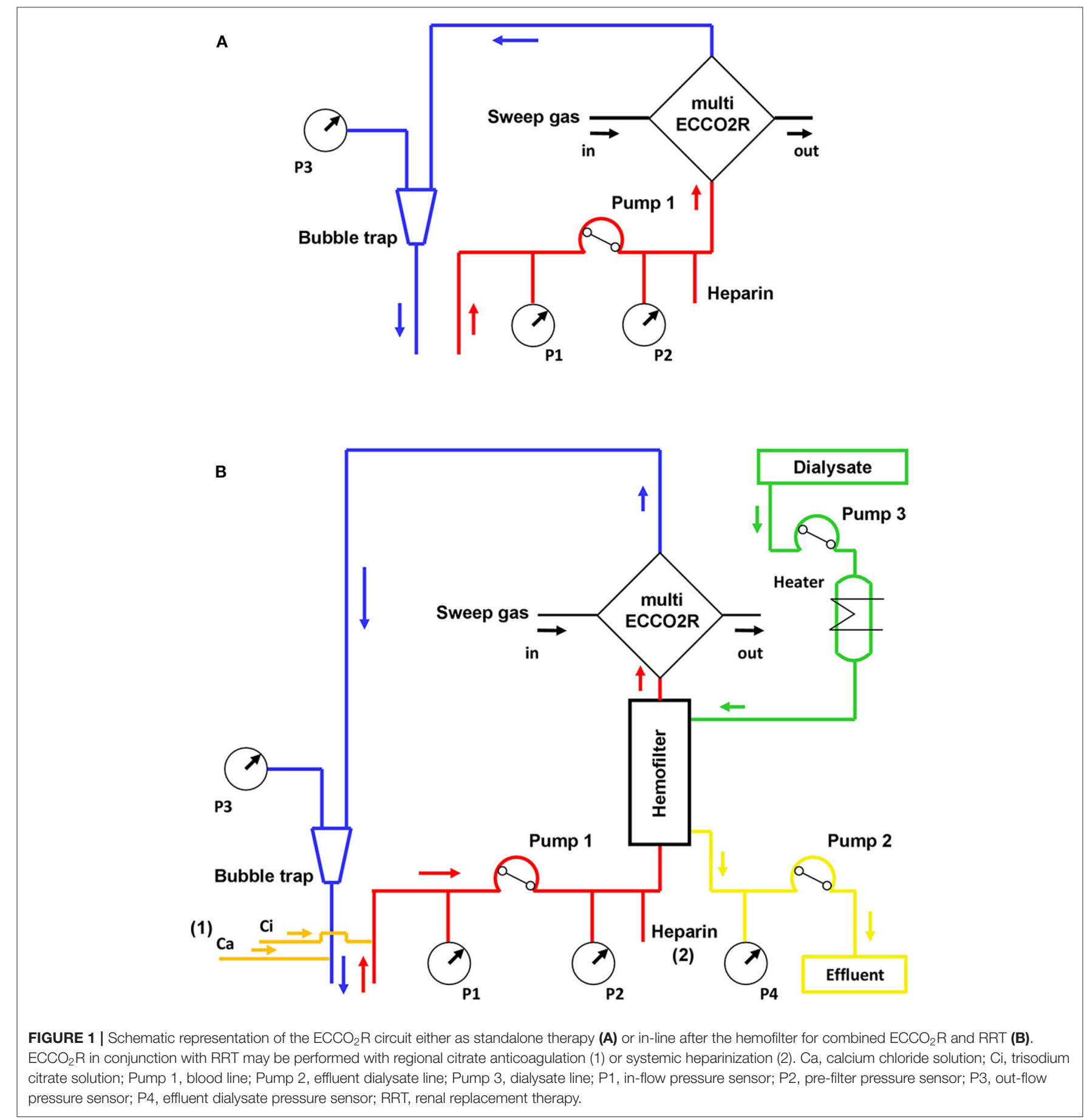


levels $(<500 \mathrm{~mL} / \mathrm{min})(5,8)$. Of note, only one study described the use of CRRT platform-driven $\mathrm{ECCO}_{2} \mathrm{R}$ without hemofilter to provide standalone $\mathrm{ECCO}_{2} \mathrm{R}$ in patients with mild to moderate ARDS. However, that trial used an $\mathrm{ECCO}_{2} \mathrm{R}$ membrane with a significantly lower surface area $\left(0.32 \mathrm{~m}^{2}\right.$ as opposed to 1.35 $\mathrm{m}^{2}$ in the current study), limiting the rate of maximal $\mathrm{CO}_{2}$ removal (9). In centers with available dialysis, low-flow $\mathrm{ECCO}_{2} \mathrm{R}$ using CRRT platforms might be an option to efficiently allocate resources for patients with hypercapnia as the main indication. The use of $\mathrm{ECCO}_{2} \mathrm{R}$ has not been described so far in COVID-19associated ARDS.

\section{MATERIALS AND METHODS}

We report results of a single-center study evaluating the feasibility and safety of $\mathrm{ECCO}_{2} \mathrm{R}$ in combination with a CRRT platform as a standalone therapy or combined with CRRT for ARDS patients with refractory hypercapnia (arterial partial pressure of $\mathrm{CO}_{2}\left[\mathrm{PaCO}_{2}\right]>55 \mathrm{mmHg}$ ) secondary to confirmed COVID-19 to effectively decrease $\mathrm{CO}_{2}$ levels and enhance lung-protective ventilation.

\section{Study Design and Participants}

COVID-19 was diagnosed according to the World Health Organization (WHO) guidance (10). All patients were nursed in an isolation intensive care unit (ICU) with other patients suffering from COVID-19. The study was prospectively registered at http://clinicaltrials.gov (Identifier: NCT04351906). Patients were sedated with fentanyl, midazolam, and propofol. Other medications, including antibiotics, fluids, catecholamines, and transfusions, were left to the discretion of the attending physician.

\section{Participants}

In-patients $\geq 18$ years of age with confirmed COVID-19 admitted to the University Hospital Giessen and Marburg, Giessen Medical Center, were enrolled in the feasibility study. Inclusion criteria were mild-to-moderate ARDS according to the Berlin definition (11), $100 \mathrm{mmHg}<$ partial alveolar oxygen pressure/fraction of inspired oxygen $\left(\mathrm{PaO}_{2} / \mathrm{FiO}_{2}\right)<300 \mathrm{mmHg}$ with positive end-expiratory pressure $>5 \mathrm{cmH}_{2} \mathrm{O}$ on mechanical ventilation expected to last $>24 \mathrm{~h}$; hypercapnia $>55 \mathrm{mmHg}$ with or without metabolic acidosis $(\mathrm{pH}<7.3)$; bilateral opacities on chest imaging; with or without AKI requiring dialysis. Exclusion criteria were age $<18$ years, pregnancy, patients with decompensated heart failure or acute coronary syndrome, respiratory acidosis with persistent partial pressure of blood carbon dioxide $\left(\mathrm{PaCO}_{2}\right)$ levels $>80 \mathrm{mmHg}$, acute brain injury, severe liver insufficiency (Child-Pugh scores $>7$ ) or fulminant hepatic failure, decision to limit therapeutic interventions, catheter access to a femoral vein or jugular vein impossible, and pneumothorax.

\section{Extracorporeal Carbon Dioxide Removal Operational Characteristic}

$\mathrm{ECCO}_{2} \mathrm{R}$ was provided using a polymethylpentene, hollow fiber, gas-exchanger membrane (multiECCO ${ }_{2} \mathrm{R}$; Eurosets,
Medolla, Italy), a labeled and certified European device to be used in conjunction with multiFiltrate CRRT platforms (Fresenius Medical Care, Bad Homburg, Germany) for combined respiratory and renal support. The manufacturer determined the multiECCO 2 R membrane's maximum duration to be $72 \mathrm{~h}$. A 13.5-Fr dual lumen hemodialysis catheter (Niagara, Bard Access, Heidelberg, Germany) was percutaneously inserted under in the femoral vein. Sweep gas flow was set at a gas/blood flow ratio of 15:1. Data were collected before starting $\mathrm{ECCO}_{2} \mathrm{R}$ (baseline) and $1,4,24$, and $48 \mathrm{~h}$ after initiation of $\mathrm{ECCO}_{2} \mathrm{R}$. A bloodline warmer (Barkey S-line) and a thermal pad (both from Barkey, Leopoldshöhe, Germany) wrapped around the multiECCO ${ }_{2} \mathrm{R}$, as well as a warming blanket, were used to avoid undercooling of the patient.

Figure 1 depicts a schematic representation of the $\mathrm{ECCO}_{2} \mathrm{R}$ setup used in this study, either as standalone therapy (Figure 1A) or in conjunction with RRT (Figure 1B). The technical terminology of the extracorporeal circuit was based on a nomenclature developed for RRT (12). For standalone $\mathrm{ECCO}_{2} \mathrm{R}$, the multiFiltrate was set in hemoperfusion mode. $\mathrm{ECCO}_{2} \mathrm{R}$ was commenced at a blood flow of $400 \mathrm{~mL} / \mathrm{min}$. Systemic heparinization was started after catheter insertion aiming for an activated partial thromboplastin time of $60-80 \mathrm{~s}$.

For $\mathrm{ECCO}_{2} \mathrm{R}+\mathrm{CRRT}$, the multiFiltrate was set in continuous venovenous hemodialysis (CVVHD) mode, and the multiECCO 2 R was inserted in series after the hemofilter (Ultraflux AV 1000S, Fresenius Medical Care, Bad Homburg, Germany). $\mathrm{ECCO}_{2} \mathrm{R}+\mathrm{CRRT}$ was commenced at a blood flow of $200 \mathrm{~mL} / \mathrm{min}$. CVVHD was delivered with an effluent dose of 25 $\mathrm{mL} / \mathrm{kg} / \mathrm{h}$ and regional citrate anticoagulation aiming a post-filter ionized calcium concentration of $\sim 0.25-0.35 \mathrm{mMol} / \mathrm{L}$.

\section{Definitions}

Lung-protective ventilation strategies were the standard of care for invasive mechanical ventilation (4). Treatment strategies for COVID-19-associated ARDS were based on the WHO interim guidance (10), which were in line with our institutional standard of care for other forms of ARDS. Of note, at the time of patient recruitment, the WHO guidance on corticosteroids to treat patients with severe and critical COVID19 was not available (13). Therefore, we did not routinely use corticosteroids for this patient population. Severe adverse events were defined as recently described (14). The feasibility of $\mathrm{ECCO}_{2} \mathrm{R}$ was assessed using Bowen et al.'s (15) feasibility framework. The use of RRT was at the discretion of the attending physician rather than by predefined biochemical or clinical criteria. However, RRT was initiated emergently when lifethreatening changes in fluid, electrolyte, and acid-base balance occurred (16). The Institute of Medical Virology (Justus Liebig University Giessen, Germany) processed nasopharyngeal swabs and bronchoalveolar lavage fluid specimens, and severe acute respiratory syndrome coronavirus 2 infection was confirmed by real-time PCR according to the previously described protocols (17). 
TABLE 1 | Characteristics of four patients with COVID-19 before $\mathrm{ECCO}_{2} \mathrm{R}$ initiation.

\begin{tabular}{|c|c|c|c|c|}
\hline & Patient 1 & Patient 2 & Patient 3 & Patient $4^{*}$ \\
\hline \multicolumn{5}{|l|}{ Demographics } \\
\hline Sex & Male & Male & Male & Male \\
\hline Age, years & 57 & 74 & 67 & 52 \\
\hline Body mass index, $\mathrm{kg} / \mathrm{m}^{2}$ & 29.4 & 24.3 & 26.8 & 42.1 \\
\hline Comorbidities & $\begin{array}{l}\text { Hypertension, } \\
\text { diabetes }\end{array}$ & $\begin{array}{l}\text { Hypertension, diabetes, } \\
\text { CAD, COPD, CKD }\end{array}$ & $\begin{array}{l}\text { Hypertension, diabetes, } \\
\text { CAD, CKD }\end{array}$ & $\begin{array}{l}\text { Hypertension, diabetes, } \\
\text { COPD, CKD }\end{array}$ \\
\hline \multicolumn{5}{|l|}{ Clinical characteristics } \\
\hline SAPS $\|$ & 37 & 51 & 54 & 43 \\
\hline SOFA score & 7 & 9 & 8 & 11 \\
\hline ICU length of stay before $\mathrm{ECCO}_{2} \mathrm{R}$ initiation, days & 23 & 6 & 25 & 8 \\
\hline \multicolumn{5}{|l|}{ Pre- $\mathrm{ECCO}_{2} \mathrm{R}$ adjuvant therapy } \\
\hline Prone positioning & Yes & Yes & Yes & Yes \\
\hline Nitric oxide & Yes & No & Yes & Yes \\
\hline Duration of $\mathrm{ECCO}_{2} \mathrm{R}$, days & 6 & 4 & 5 & 8 \\
\hline$V_{T}, \mathrm{~mL} / \mathrm{kg}$ PBW & 5.6 & 7.2 & 6.5 & 7.3 \\
\hline $\mathrm{RR}$, breaths/min & 30 & 19 & 31 & 21 \\
\hline$V_{E}, L / m i n$ & 10.3 & 10.1 & 10.5 & 13.8 \\
\hline P PLAT, $\mathrm{cmH}_{2} \mathrm{O}$ & 30 & 26 & 31 & 27 \\
\hline PEEP, $\mathrm{cmH}_{2} \mathrm{O}$ & 10 & 11 & 6 & 11 \\
\hline Driving pressure, $\mathrm{cmH}_{2} \mathrm{O}$ & 20 & 15 & 25 & 16 \\
\hline Compliance, $\mathrm{mL} / \mathrm{mbar}$ & 18.4 & 34.6 & 18.4 & 41.2 \\
\hline $\mathrm{PaO}_{2} / \mathrm{FiO}_{2}$ ratio & 153.3 & 150.6 & 160.0 & 140.0 \\
\hline $\mathrm{PaCO}_{2}, \mathrm{mmHg}$ & 57.4 & 70.0 & 56.6 & 58.7 \\
\hline $\mathrm{pH}$ & 7.38 & 7.29 & 7.41 & 7.23 \\
\hline Arterial $\mathrm{HCO}_{3^{-}}, \mathrm{mMol} / \mathrm{L}$ & 33.3 & 32.3 & 35.1 & 21.4 \\
\hline LVEF, \% & 60 & 40 & 65 & 60 \\
\hline Norepinephrine dose, $\mu \mathrm{g} / \mathrm{kg} / \mathrm{min}$ & 0.002 & 0.336 & 0.219 & 0.038 \\
\hline \multicolumn{5}{|l|}{ Laboratory findings } \\
\hline White cell count, g/L & 7.1 & 12.9 & 26.3 & 17.1 \\
\hline Total lymphocytes & 1.54 & 0.94 & 1.94 & 1.26 \\
\hline Hemoglobin, g/dL & 84 & 95 & 90 & 93 \\
\hline Platelet count, giga/L & 316 & 357 & 288 & 301 \\
\hline Creatinine, $\mathrm{mg} / \mathrm{dL}^{\dagger}$ & 0.5 & 1.9 & 1.0 & 2.0 \\
\hline Urea, mg/dL ${ }^{\ddagger}$ & 37 & 197 & 101 & 230 \\
\hline Lactate dehydrogenase, U/L & 311 & 492 & 237 & 365 \\
\hline Alanine aminotransferase, $U / L$ & 104 & 378 & 40 & 212 \\
\hline Aspartate aminotransferase, $\mathrm{U} / \mathrm{L}$ & 44 & 359 & 35 & 363 \\
\hline Albumin, $g / L$ & 24.9 & 23.6 & 29.2 & 29.7 \\
\hline B-type natriuretic peptide, $\mathrm{pg} / \mathrm{mL}$ & 48 & 591 & 93 & 9 \\
\hline C-reactive protein, mg/L & 71.1 & 164.5 & 113.9 & 197.7 \\
\hline Procalcitonin, $\mu \mathrm{g} / \mathrm{L}$ & 0.5 & 6.1 & 7.5 & 1.6 \\
\hline Interleukin-6, $\mu \mathrm{g} / \mathrm{L}$ & 74 & 2150 & 95 & 55 \\
\hline Ferritin, $\mu \mathrm{g} / \mathrm{L}$ & 1588 & 2107 & 723 & 1076 \\
\hline D-dimer, mg/L & 3.1 & 15.3 & 1.77 & 3.9 \\
\hline
\end{tabular}

${ }^{*}$ Patient received $E C \mathrm{COO}_{2} R+C R R T$.

${ }^{\dagger}$ To convert the values for serum creatinine to $\mathrm{mg} / \mathrm{dL}$, multiply by 88.4 .

${ }^{\ddagger}$ To convert the value for urea to blood urea nitrogen, multiply by 0.467 .

CAD, coronary artery disease; CKD, chronic kidney disease; COPD, chronic obstructive pulmonary disease; COVID-19, coronavirus disease 2019; CRRT, continuous renal replacement therapy; $\mathrm{ECCO}_{2} \mathrm{R}$, extracorporeal carbon dioxide removal; $\mathrm{FiO}_{2}$, fraction of inspired oxygen; $\mathrm{HCO}_{3}^{-}$, bicarbonate; ICU, intensive care unit; LVEF, left ventricular ejection fraction; NA, not applicable/not available; $\mathrm{PaCO}_{2}$, arterial partial pressure of carbon dioxide; $\mathrm{PaO}_{2}$, arterial partial pressure of oxygen; PBW, predicted body weight; PEEP, positive end-expiratory pressure; PPLAT, plateau pressure; RR, respiratory rate; SAP, Simplified Acute Physiology Score; SOFA, Sequential Organ Failure Assessment; $V_{E}$, minute volume; $V_{T}$, tidal volume. 
TABLE 2 | Individual time course of operational characteristics, blood gas, ventilatory, and hemodynamic parameters during $\mathrm{ECCO}_{2} \mathrm{R}$.

\begin{tabular}{|c|c|c|c|c|c|c|c|c|c|c|c|c|c|c|c|c|c|c|c|c|}
\hline \multirow[b]{2}{*}{ Operational characteristics } & \multicolumn{5}{|c|}{ Patient 1} & \multicolumn{5}{|c|}{ Patient 2} & \multicolumn{5}{|c|}{ Patient 3} & \multicolumn{5}{|c|}{ Patient $4^{*}$} \\
\hline & Baseline & $1 \mathrm{~h}$ & $4 \mathrm{~h}$ & $24 \mathrm{~h}$ & $48 \mathrm{~h}$ & Baseline & $1 \mathrm{~h}$ & $4 \mathrm{~h}$ & $24 \mathrm{~h}$ & $48 \mathrm{~h}$ & Baseline & $1 \mathrm{~h}$ & $4 \mathrm{~h}$ & $24 \mathrm{~h}$ & $48 \mathrm{~h}$ & Baseline & $1 \mathrm{~h}$ & $4 \mathrm{~h}$ & $24 \mathrm{~h}$ & $48 \mathrm{~h}$ \\
\hline Blood flow, $\mathrm{mL} / \mathrm{min}$ & 400 & 400 & 400 & 400 & 400 & 400 & 400 & 400 & 400 & 400 & 400 & 400 & 400 & 400 & 400 & 200 & 200 & 200 & 200 & 200 \\
\hline Sweep gas flow, L/min & 6 & 6 & 6 & 6 & 6 & 6 & 6 & 6 & 6 & 6 & 6 & 6 & 6 & 6 & 6 & 3.5 & 3.5 & 3.5 & 3.5 & 3.5 \\
\hline CRRT ultrafiltration rate, $\mathrm{mL} / \mathrm{h}$ & NA & NA & NA & NA & NA & NA & NA & NA & NA & NA & NA & NA & NA & NA & NA & 0 & 0 & 0 & 0 & $100^{+}$ \\
\hline CRRT effluent rate, $\mathrm{mL} / \mathrm{kg} / \mathrm{h}$ & NA & NA & NA & NA & NA & NA & NA & NA & NA & NA & NA & NA & NA & NA & NA & 25 & 25 & 25 & 25 & 25 \\
\hline aPTT, s & 30 & NA & 67 & 98 & 93 & 48 & 80 & 79 & 79 & NA & 85 & NA & NA & 56 & 65 & 27 & 26 & NA & 26 & $25 \ddagger$ \\
\hline \multicolumn{21}{|l|}{ Blood gas parameters } \\
\hline \multicolumn{21}{|l|}{ Arterial } \\
\hline $\mathrm{PaCO}_{2}, \mathrm{mmHg}$ & 57.4 & 43.5 & 43.0 & 38.3 & 42.4 & 70.0 & 50.0 & 54.7 & 53.1 & 52.8 & 56.6 & 42.1 & 42.6 & 42.4 & 46.5 & 58.7 & 46.5 & 46.8 & 47.2 & 46.3 \\
\hline $\mathrm{pH}$ & 7.38 & 7.48 & 7.48 & 7.53 & 7.47 & 7.29 & 7.44 & 7.38 & 7.39 & 7.35 & 7.41 & 7.53 & 7.51 & 7.50 & 7.47 & 7.23 & 7.30 & 7.36 & 7.38 & 7.40 \\
\hline $\mathrm{PaO}_{2}, \mathrm{mmHg}$ & 69.2 & 63.0 & 71.0 & 71.0 & 66.0 & 68.0 & 66.0 & 69.0 & 74.0 & 62.0 & 80.0 & 65.0 & 79.0 & 82.0 & 79.0 & 77.0 & 81.0 & 89.0 & 71.0 & 94.0 \\
\hline $\mathrm{HCO}_{3^{-}}, \mathrm{mMol} / \mathrm{L}$ & 33.3 & 32.0 & 31.6 & 31.9 & 30.8 & 32.3 & 33.1 & 32.3 & 32.4 & 28.1 & 35.1 & 34.6 & 33.8 & 33.0 & 33.3 & 21.4 & 21.9 & 25.9 & 27.2 & 27.8 \\
\hline $\mathrm{BE}, \mathrm{mMol} / \mathrm{L}$ & 7.6 & 8.3 & 7.9 & 8.7 & 6.9 & 4.8 & 8.2 & 6.2 & 6.6 & 2.5 & 9.4 & 10.8 & 10.2 & 9.1 & 8.9 & -5.5 & -3.9 & 0.8 & 2.3 & 3.0 \\
\hline \multicolumn{21}{|l|}{ Pre-ECCO ${ }_{2} \mathrm{R}$} \\
\hline $\mathrm{PCO}_{2}, \mathrm{mmHg}$ & NA & 54.2 & 53.2 & 42.5 & 55.9 & NA & 58.3 & 57.0 & 55.1 & 52.8 & NA & 52.4 & 50.0 & 51.2 & 54.0 & NA & 49.8 & 41.4 & 44.5 & 48.7 \\
\hline $\mathrm{HCO}_{3^{-}}, \mathrm{mMol} / \mathrm{L}$ & NA & 33.9 & 33.2 & 33.4 & 31.8 & NA & 34.5 & 31.4 & 30.6 & 25.7 & NA & 33.5 & 34.7 & 35.0 & 35.7 & NA & 20.7 & 24.9 & 27.2 & 28.7 \\
\hline $\mathrm{BE}, \mathrm{mMol} / \mathrm{L}$ & NA & 8.4 & 7.9 & 9.6 & 6.2 & NA & 8.6 & 8.0 & 7.2 & 0.0 & NA & 12.0 & 12.0 & 10.0 & 10.5 & NA & -5.9 & 0.5 & 2.6 & 3.6 \\
\hline \multicolumn{21}{|l|}{ Post-ECCO ${ }_{2} \mathrm{R}$} \\
\hline $\mathrm{PCO}_{2}, \mathrm{mmHg}$ & NA & 15.0 & 13.4 & 14.1 & 18.6 & NA & 14.3 & 12.5 & 13.6 & 11.9 & NA & 8.2 & 10.7 & 12.4 & 15.7 & NA & 7.6 & 9.5 & 11.3 & 8.5 \\
\hline $\mathrm{HCO}_{3^{-}}, \mathrm{mMol} / \mathrm{L}$ & NA & 32.3 & 29.5 & 30.3 & 27.4 & NA & 27.2 & 25.6 & 26.3 & 24.6 & NA & 29.0 & 34.9 & 28.5 & 29.9 & NA & 9.6 & 15.8 & 17.0 & 17.1 \\
\hline $\mathrm{BE}, \mathrm{mMol} / \mathrm{L}$ & NA & 8.3 & 10.5 & 11.1 & 7.4 & NA & 10.2 & 7.7 & 7.9 & 0.2 & NA & 11.4 & 11.1 & 11.2 & 11.2 & NA & -9.5 & -1.8 & -1.1 & 0.6 \\
\hline \multicolumn{21}{|l|}{ Ventilator parameters } \\
\hline $\mathrm{V}_{T}, \mathrm{~mL} / \mathrm{kg}$ PBW & 5.6 & 5.6 & 5.3 & 5.3 & 5.2 & 7.2 & 7.2 & 7.4 & 6.3 & 6.4 & 6.5 & 5.9 & 5.6 & 4.4 & 4.9 & 7.3 & 7.4 & 7.4 & 7.2 & 7.0 \\
\hline $\mathrm{RR}$, breaths/min & 30 & 30 & 28 & 26 & 24 & 19 & 19 & 18 & 18 & 18 & 31 & 30 & 30 & 30 & 26 & 21 & 21 & 21 & 21 & 21 \\
\hline$V_{E}, L / m i n$ & 10.3 & 10.6 & 10.8 & 10.7 & 7.5 & 10.1 & 9.5 & 9.2 & 8.6 & 9.4 & 10.5 & 11.5 & 10.0 & 9.2 & 6.2 & 13.8 & 13.8 & 13.9 & 13.7 & 13.4 \\
\hline P PLAT, $\mathrm{CmH}_{2} \mathrm{O}$ & 30 & 30 & 29 & 22 & 22 & 26 & 26 & 25 & 24 & 24 & 31 & 31 & 31 & 30 & 28 & 27 & 27 & 27 & 26 & 25 \\
\hline PEEP, $\mathrm{cmH}_{2} \mathrm{O}$ & 10 & 10 & 9 & 8 & 8 & 11 & 11 & 11 & 11 & 11 & 6 & 6 & 6 & 6 & 6 & 11 & 11 & 11 & 11 & 11 \\
\hline Driving pressure, $\mathrm{cmH}_{2} \mathrm{O}$ & 20 & 20 & 20 & 14 & 14 & 15 & 15 & 14 & 13 & 13 & 25 & 25 & 25 & 24 & 22 & 16 & 16 & 16 & 15 & 14 \\
\hline Compliance, $\mathrm{mL} / \mathrm{mbar}$ & 18.4 & 18.5 & 17.5 & 24.9 & 24.5 & 34.6 & 32.5 & 27.1 & 33.2 & 31.4 & 18.4 & 15.8 & 14.3 & 13.7 & 13.6 & 41.2 & 41.4 & 41.5 & 43.5 & 45.6 \\
\hline $\mathrm{PaO}_{2} / \mathrm{FiO}_{2}$ ratio & 153.3 & 153.3 & 157.8 & 157.8 & 165.0 & 150.6 & 132.7 & 153.3 & 160.0 & 157.8 & 160.0 & 130.0 & 143.6 & 136.7 & 134.0 & 140.0 & 147.3 & 161.8 & 157.8 & 175.1 \\
\hline \multicolumn{21}{|l|}{ Hemodynamic parameters } \\
\hline Mean arterial pressure, mmHg & 71 & 74 & 74 & 76 & 75 & 64 & 89 & 82 & 69 & 66 & 79 & 79 & 77 & 74 & 66 & 63 & 62 & 66 & 67 & 77 \\
\hline Heart rate, beats/min & 93 & 83 & 84 & 92 & 78 & 70 & 58 & 66 & 72 & 70 & 105 & 95 & 92 & 90 & 88 & 84 & 83 & 76 & 72 & 86 \\
\hline Norepinephrine dose, $\mu \mathrm{g} / \mathrm{kg} / \mathrm{min}$ & 0.002 & 0.002 & 0.002 & 0.002 & 0.002 & 0.336 & 0.336 & 0.420 & 0.428 & 0.430 & 0.219 & 0.274 & 0.192 & 0.205 & 0.207 & 0.038 & 0.038 & 0.038 & 0.038 & 0.038 \\
\hline
\end{tabular}

\section{*Patient received $\mathrm{ECCO}_{2} R+\mathrm{CRRT}$}

${ }^{\dagger} \mathrm{CRRT}$ ultrafiltration was started at $38 \mathrm{~h}$ post- $E \mathrm{CCO}_{2}$ R initiation.

${ }^{\ddagger} E C C_{2} R+C R R T$ was performed with regional citrate anticoagulation.

aPTT, activated partial thromboplastin time; $B E$, base excess; COVID-19, coronavirus disease 2019; $C R R T$, continuous renal replacement therapy; $E C C \mathrm{C}_{2} R$, extracorporeal carbon dioxide removal; FiO 2 , fraction of inspired oxygen; $\mathrm{HCO}_{3-}$, bicarbonate; $\mathrm{PaCO}_{2}$, arterial partial pressure of carbon dioxide; $\mathrm{PCO}_{2}$, venous partial pressure of carbon dioxide; $\mathrm{PaO}_{2}$, arterial partial pressure of oxygen; $P B W$, predicted body weight; $P E E P$, positive end-expiratory pressure; $P_{P L A T}$, plateau pressure; $R C A$, regional citrate anticoagulation; $R R$, respiratory rate; $N A$, not applicable/not available; $V_{E}$, minute volume; $V_{T}$, tidal volume. 


\section{RESULTS}

We report data of four male patients (median age: 62 [range, 52-74] years) admitted to our ICU between April and May 2020 due to ARDS secondary to confirmed severe acute respiratory syndrome coronavirus 2 infection (for clinical data, see Table 1). After implementing adjunctive measures for ARDS, all patients showed an improvement in oxygenation $\left(\mathrm{PaO}_{2} / \mathrm{FiO}_{2}\right.$ ratio); however, in the later course of intensive care, all patients developed severe hypercapnia despite escalated ventilation parameters. In patients 1 and 3, hypercapnia was seen as the result of diffuse consolidations and fibrotic remodeling of the lungs as indicated by the low compliance (18.4 $\mathrm{mL} / \mathrm{mbar}$ ), whereas patients 2 and 4 developed hypercapnia, at least in part, secondary to underlying chronic obstructive pulmonary disease.

$\mathrm{ECCO}_{2} \mathrm{R}$ was implemented at a blood-flow rate of $400 \mathrm{~mL} / \mathrm{min}$ in patients $1-3$, resulting in a $\mathrm{PaCO}_{2}$ decrease from a median 57.4 [56.6-70.0] to 43.5 [42.1-50.0] $\mathrm{mmHg}$ within $1 \mathrm{~h}$, whereas $\mathrm{pH}$ increased from a median 7.38 [7.297.41] to 7.48 [7.44-7.53] $\mathrm{mmHg}$ within $1 \mathrm{~h}$ (Table 2). Patient 4 developed combined respiratory and metabolic acidosis secondary to hypercapnia and $\mathrm{AKI}$, and $\mathrm{ECCO}_{2} \mathrm{R}+\mathrm{CRRT}$ was commenced with a blood-flow rate of $200 \mathrm{~mL} / \mathrm{min}$, leading to a decrease of $\mathrm{PaCO}_{2}$ from 58.7 to $46.5 \mathrm{mmHg}$ within $1 \mathrm{~h}$ while $\mathrm{pH}$ and bicarbonate levels progressively increased. CRRT ultrafiltration $(100 \mathrm{~mL} / \mathrm{h})$ was started at $38 \mathrm{~h}$ post- $\mathrm{ECCO}_{2} \mathrm{R}$ initiation due to oliguria. Tidal volume, plateau and driving pressure, as well as respiratory rate could be reduced during the second day of $\mathrm{ECCO}_{2} \mathrm{R}$ (from median 6.9 [5.6-7.3] to $5.8[4.9-7.0] \mathrm{mL} / \mathrm{kg}$ PBW, median 28.5 [26.0-31.0] to 24.5 [22.0-28.0] $\mathrm{cmH}_{2} \mathrm{O}$, median 18.0 [15.0-25.0] to 14.0 [13.0-22.0] $\mathrm{cmH}_{2} \mathrm{O}$, and median $25.5[19.0-31.0]$ to 21.5 [18.0-26.0] breaths/min, respectively; Figure 2A). The $\mathrm{PaO}_{2} / \mathrm{FiO}_{2}$ ratio remained unchanged throughout the study period (from median 152.0 [140.0-160.0] to 161.4 [134.0-175.1]). There was no detectable impact of $\mathrm{ECCO}_{2} \mathrm{R}$ on hemodynamics and vasopressor support. A comparison of pre- and post- $\mathrm{ECCO}_{2} \mathrm{R}$ $\mathrm{PCO}_{2}$ values showed a $\sim 30 \mathrm{mmHg}$ decrease (Figure 2B). No patient- or $\mathrm{ECCO}_{2} \mathrm{R} / \mathrm{CRRT}$-related adverse events occurred. Downtime ranged from 2 to $8 \%$ of the total treatment time owing due to the turning of patients into the prone position. In all four patients, the $\mathrm{ECCO}_{2} \mathrm{R}$ treatment could be terminated after a median of 5.5 (4.5-7.5) days due to a sustained improvement in hypercapnia. In patient 4 , however, CRRT was continued for another 4 days due to oliguria. Furthermore, patient 2 developed AKI stage 3, necessitating CRRT 6 days after the termination of $\mathrm{ECCO}_{2} \mathrm{R}$ as a sequel to septic shock.

\section{DISCUSSION}

Our data indicate that low-flow $\mathrm{ECCO}_{2} \mathrm{R}$ using CRRT platforms might be safe and feasible to provide either standalone $\mathrm{ECCO}_{2} \mathrm{R}$ or $\mathrm{ECCO}_{2} \mathrm{R}$ combined with CRRT. This minimally invasive approach leads to efficient $\mathrm{CO}_{2}$ removal in the setting of moderate ARDS. No patient- or ECCO2R/CRRT-related adverse events occurred. Importantly, these data also implicate that

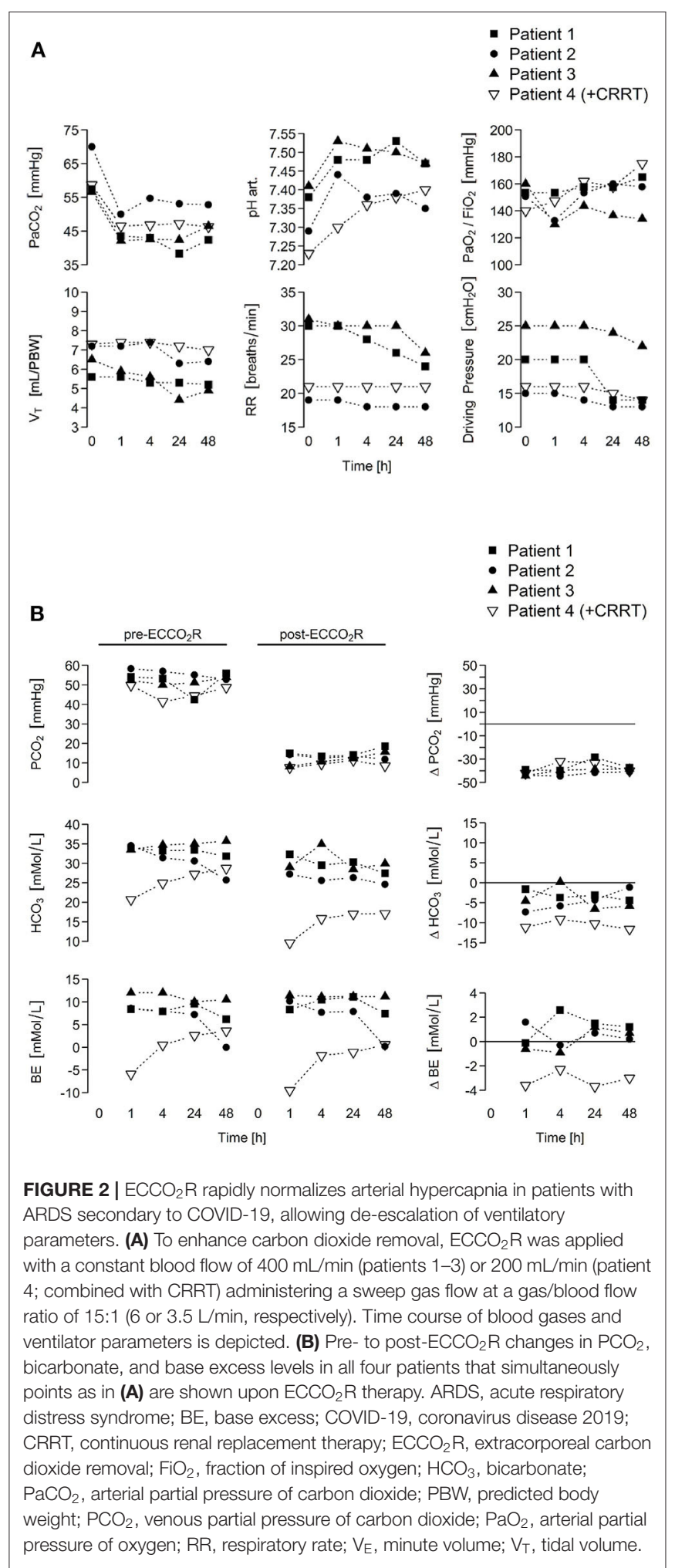

every ICU with available dialysis may apply RRT platformdriven $\mathrm{ECCO}_{2} \mathrm{R}$ to limit ventilator-induced lung injury or rescue uncontrollable respiratory acidosis even in situations where 
"standard" $\mathrm{ECCO}_{2} \mathrm{R}$ consoles are not available. To the best of our knowledge, this is the first description of $\mathrm{ECCO}_{2} \mathrm{R}$ in COVID-19. Although these data may provide the rationale for randomized clinical trials, the following limitations need to be acknowledged. Given the invasive nature of an $\mathrm{ECCO}_{2} \mathrm{R}$ therapy, future randomized trials are required to assess the overall benefit and harm before widespread implementation can be recommended. Also, eligibility criteria should be further examined, particularly in those without an indication for CRRT. Furthermore, if the $\mathrm{ECCO}_{2} \mathrm{R}$ is intended to be continuous, sustaining a blood flow of $400 \mathrm{~mL} / \mathrm{min}$ with a temporary catheter may be challenging, particularly in patients with COVID-19 who are obese or require prone positioning. COVID-19 induces a hypercoagulable state in many patients, which may result in premature extracorporeal circuit failure (18). No studies are available to date to aid in the selection of anticoagulation strategy, in particular when introducing an extracorporeal circulation. Thus, close monitoring of the extracorporeal circuit performance is advisable to ensure maximal circuit patency, as the initial anticoagulation strategy may not be effective in all patients, and a stepwise escalation and/or alternative plans (e.g., combination of different anticoagulation strategies) may be required. However, if using CRRT, we suggest CVVHD or continuous venovenous hemodiafiltration to decrease filtration fraction and reduce the risk of circuit clotting (19). In addition, COVID-19associated ARDS may follow uncontrolled host immune response to the virus with the release of various immune mediators, especially cytokines, damage-associated molecular patterns, and pathogen-associated molecular patterns $(20,21)$. Extracorporeal blood purification techniques (e.g., hemoperfusion; RRT with surface-modified AN69, polymethylmethacrylate, or high-cut off membranes) have been proposed as adjuvant therapy for critically ill patients with COVID-19 to restore immune homeostasis through the removal of these circulating mediators (7). As many healthcare agencies have authorized emergency use of various extracorporeal blood purification techniques, these treatments might be indicated as sequential extracorporeal therapies in special cases in which immuno-dysregulation is evident, inflammatory parameters or cytokines are elevated, and other supportive therapies are failing or insufficient. Nonetheless, careful patient selection is required if these are to be used, as the benefits and adverse effects in COVID19 patients have not been formally studied. Finally, additional costs associated with the use of $\mathrm{ECCO}_{2} \mathrm{R}$ in conjunction with RRT platforms in COVID-19-associated ARDS may be offset by a potential cost reduction through the elimination of daily rental costs for standalone $\mathrm{ECCO}_{2} \mathrm{R}$ consoles, the recruitment of dialysis professionals in centers with available dialysis to operate $\mathrm{ECCO}_{2} \mathrm{R}$, and a shorter length of ICU and hospital stay. However, large, multicenter randomized clinical trials are required to support the cost-benefit ratio of $\mathrm{ECCO}_{2} \mathrm{R}$ in conjunction with RRT platforms.

In conclusion, our data indicate that low-flow $\mathrm{ECCO}_{2} \mathrm{R}$ using CRRT platforms might be safe and feasible to provide either standalone $\mathrm{ECCO}_{2} \mathrm{R}$ or $\mathrm{ECCO}_{2} \mathrm{R}$ combined with CRRT. A multicenter randomized trial is warranted to assess the effects of CRRT platform-driven $\mathrm{ECCO}_{2} \mathrm{R}$ on clinical outcomes of patients with ARDS secondary to COVID-19 or other pathogenic factors.

\section{DATA AVAILABILITY STATEMENT}

The datasets used and/or analyzed during the study are available from the corresponding author upon reasonable request.

\section{ETHICS STATEMENT}

The study protocol was approved by the Ethics Committee of the Medical Faculty of the Justus Liebig University Giessen (AZ 63/20) and complied with the Declaration of Helsinki. Legally authorized representatives of the patients provided written informed consent.

\section{AUTHOR CONTRIBUTIONS}

FH-S, H-WB, CR, H-DW, WS, and IV: concept and design of the study. VR, BK, SK, KT, MH, RM, SH, and OK: literature research and clinical advice. FH-S, H-WB, JW, CR, VR, BK, SK, KT, MH, RM, SH, OK, H-DW, WS, and IV: acquisition, analyses, or interpretation of data, and critical revision of the manuscript for important intellectual content. FH-S and IV: manuscript drafting and had full access to all study data and had final responsibility for submitting for publication. FH-S, JW, and IV: figure illustration. HWB, H-DW, WS, and IV: study supervision. All authors shared the study design, data collection, data analyses, data interpretation, as well as preparation, review, and approval of the manuscript.

\section{FUNDING}

This work was supported by grants from the Federal Ministry of Education and Research [German Centre for Lung Research (DZL/ALI); to SH, WS, and IV], the German Research Foundation (DFG/KFO309; to JW, RM, $\mathrm{SH}$, WS, and IV), The Cardio-Pulmonary Institute (EXC 2026; Project ID: 390649896; to JW, KT, RM, SH, WS, and IV), and the von Behring Röntgen Foundation (Project 66-LV07; to IV).

\section{ACKNOWLEDGMENTS}

The authors thank the medical and nursing staff of the ICU for their hard work and commitment to patient wellbeing. Without their support, this work would not have been possible. 


\section{REFERENCES}

1. Wang D, Hu B, Hu C, Zhu F, Liu X, Zhang J, et al. Clinical characteristics of 138 hospitalized patients with 2019 novel coronavirus-infected pneumonia in Wuhan, China. JAMA. (2020) 323:1061-9. doi: 10.1001/jama.2020.1585

2. Yang X, Yu Y, Xu J, Shu H, Xia J, Liu H, et al. Clinical course and outcomes of critically ill patients with SARS-CoV-2 pneumonia in Wuhan, China: a single-centrered, retrospective, observational study. Lancet Respir Med. (2020) 8:475-81. doi: 10.1016/S2213-2600(20)30079-5

3. Arentz M, Yim E, Klaff L, Lokhandwala S, Riedo FX, Cong M, et al. Characteristics and outcomes of 21 critically ill patients with COVID19 in Washington State. JAMA. (2020) 323:1612-4. doi: 10.1001/jama.202 0.4326

4. Fan E, Del Sorbo L, Goligher EC, Hodgson CL, Munshi L, Walkey AJ, et al. An Official American Thoracic Society/European Society of Intensive Care Medicine/Society of Critical Care Medicine clinical practice guideline: mechanical ventilation in adult patients with acute respiratory distress syndrome. Am J Respir Crit Care Med. (2017) 195:125363. doi: 10.1164/rccm.201703-0548ST

5. Fanelli V, Cantaluppi V, Alessandri F, Costamagna A, Capello P, Brazzi L, et al. Extracorporeal $\mathrm{CO}_{2}$ removal may improve renal function of patients with acute respiratory distress syndrome and acute kidney injury: an openlabel, interventional clinical trial. Am J Respir Crit Care Med. (2018) 198:68790. doi: 10.1164/rccm.201712-2575LE

6. Nin N, Muriel A, Penuelas O, Brochard L, Lorente JA, Ferguson ND, et al. Severe hypercapnia and outcome of mechanically ventilated patients with moderate or severe acute respiratory distress syndrome. Intens Care Med. (2017) 43:200-8. doi: 10.1007/s00134-016-4611-1

7. Ronco C, Reis T, Husain-Syed F. Management of acute kidney injury in patients with COVID-19. Lancet Respir Med. (2020) 8:738-42. doi: 10.1016/s2213-2600(20)30229-0

8. Forster C, Schriewer J, John S, Eckhardt KU, William C. Low-flow $\mathrm{CO}_{2}$ removal integrated into a renal-replacement circuit can reduce acidosis and decrease vasopressor requirements. Crit Care. (2013) 17:R154. doi: 10.1186/cc12833

9. Schmidt M, Jaber S, Zogheib E, Godet T, Capellier G, Combes A. Feasibility and safety of low-flow extracorporeal $\mathrm{CO}_{2}$ removal managed with a renal replacement platform to enhance lung-protective ventilation of patients with mild-to-moderate ARDS. Crit Care. (2018) 2022:122. doi: 10.1186/s13054-018-2038-5

10. World Health Organization. Clinical Management of Severe Acute Respiratory Infection (SARI) When COVID-19 Disease Is Suspected. Interim Guidance (2020). Available online at: https://www.who.int/docs/default-source/ coronaviruse/clinical-management-of-novel-cov.pdf (accessed August 24, 2020).

11. ARDS Definition Task Force Force, Ranieri VM, Rubenfeld GD, Thompson BT, Ferguson ND, Caldwell E, et al. Acute respiratory distress syndrome: the Berlin definition. JAMA. (2012) 307:2526-33. doi: 10.1001/jama.20 12.5669

12. Villa G, Neri M, Bellomo R, Cerda J, De Gaudio AR, De Rosa S, et al. Nomenclature Standardization Initiative (NSI) Alliance. Nomenclature for renal replacement therapy and blood purification techniques in critically ill patients: practical applications. Crit Care. (2016) 20:283. doi: 10.1186/s13054-016-1456-5

13. World Health Organization. Corticosteroids for COVID-19. Living Guidance (2020). Available online at: https://www.who.int/publications/i/item/WHO2019-nCoV-Corticosteroids-2020.1

14. Combes A, Fanelli V, Pham T, Ranieri VM, European Society of Intensive Care Medicine Trials Group, Strategy of Ultra-Protective Lung Ventilation With Extracorporeal $\mathrm{CO}_{2}$ Removal for New-Onset Moderate to severe ARDS (SUPERNOVA) Investigators. Feasibility and safety of extracorporeal $\mathrm{CO}_{2}$ removal to enhance protective ventilation in acute respiratory distress syndrome: the SUPERNOVA study. Intens Care Med. (2019) 45:592600. doi: 10.1007/s00134-019-05567-4

15. Bowen DJ, Kreuter M, Spring B, Cofta-Woerpel L, Linnan L, Weiner D, et al. How we design feasible studies. Am J Prev Med. (2009) 36:4527. doi: 10.1016/j.amepre.2009.02.002

16. Kidney Disease: Improving Global Outcomes (KDIGO) Acute Kidney Injury Work Group. KDIGO clinical practice guideline for acute kidney injury. Kidney Int Suppl. (2012) 2:1-138.

17. Corman VM, Landt O, Kaiser M, Molenkamp R, Meijer A, Chu DK, et al. Detection of 2019 novel coronavirus (2019-nCoV) by real-time RT-PCR. Euro Surveill. (2020) 25:2000045. doi: 10.2807/1560-7917.ES.2020.25.3.2000045

18. Iba T, Levy JH, Levi M, Connors JM, Thachil J. Coagulopathy of coronavirus disease 2019. Crit Care Med. (2020) 48:135864. doi: 10.1097/CCM.0000000000004458

19. Joannidis M, Oudemans-van Straaten HM. Clinical review: patency of the circuit in continuous renal replacement therapy. Crit Care. (2007) 11:218. doi: 10.1186/cc5937

20. Huang C, Wang Y, Li X, Ren L, Zhao J, Hu Y, et al. Clinical features of patients infected with 2019 novel coronavirus in Wuhan, China. Lancet. (2020) 395:497-506. doi: 10.1016/S0140-6736(20)30183-5

21. Ruan Q, Yang K, Wang W, Jiang L, Song J. Clinical predictors of mortality due to COVID-19 based on an analysis of data of 150 patients from Wuhan, China. Intens Care Med. (2020) 46:846-8. doi: 10.1007/s00134-02005991-x

Conflict of Interest: WS received personal fees for consulting from Bayer Pharma, Liquidia Technologies, and United Therapeutics outside the submitted work.

The remaining authors declare that the research was conducted in the absence of any commercial or financial relationships that could be construed as a potential conflict of interest.

Copyright (c) 2020 Husain-Syed, Birk, Wilhelm, Ronco, Ranieri, Karle, Kuhnert, Tello, Hecker, Morty, Herold, Kehl, Walmrath, Seeger and Vadász. This is an openaccess article distributed under the terms of the Creative Commons Attribution License (CC BY). The use, distribution or reproduction in other forums is permitted, provided the original author(s) and the copyright owner(s) are credited and that the original publication in this journal is cited, in accordance with accepted academic practice. No use, distribution or reproduction is permitted which does not comply with these terms. 